\title{
LIX1 regulates YAP1 activity and controls the proliferation and differentiation of stomach mesenchymal progenitors
}

\author{
Jennifer McKey, Delphine Martire, Pascal de Santa Barbara ${ }^{\dagger}$ and Sandrine Faure ${ }^{*+}$
}

\begin{abstract}
Background: Smooth muscle cell (SMC) plasticity maintains the balance between differentiated SMCS and proliferative mesenchymal progenitors, crucial for muscular tissue homeostasis. Studies on the development of mesenchymal progenitors into SMCs have proven useful in identifying molecular mechanisms involved in digestive musculature plasticity in physiological and pathological conditions.

Results: Here, we show that Limb Expression 1 (LIXI) molecularly defines the population of mesenchymal progenitors in the developing stomach. Using in vivo functional approaches in the chick embryo, we demonstrate that LIXI is a key regulator of stomach $S M C$ development. We show that $L I X 1$ is required for stomach SMC determination to regulate the expression of the pro-proliferative gene YAP1 and mesenchymal cell proliferation. However, as stomach development proceeds, sustained $L I X 1$ expression has a negative impact on further SMC differentiation and this is associated with a decrease in YAP1 activity.

Conclusions: We demonstrate that expression of LIX 1 must be tightly regulated to allow fine-tuning of the transcript levels and state of activation of the pro-proliferative transcriptional coactivator YAP1 to regulate proliferation rates of stomach mesenchymal progenitors and their differentiation. Our data highlight dual roles for LIX1 and YAP1 and provide new insights into the regulation of cell density-dependent proliferation, which is essential for the development and homeostasis of organs.
\end{abstract}

Keywords: Gastrointestinal tract, Mesenchymal progenitors, Smooth muscle cells, LIX1, YAP1, FGF pathway, Density-dependent cell proliferation

\section{Background}

The gastrointestinal (GI) tract is a vital organ, highly conserved across vertebrate species and essential for the absorption of water and nutrients. During development, the GI tract arises from a primary uniform tube composed of mesoderm and endoderm. The mesoderm gives rise to the digestive mesenchyme, which in turn differentiates into multiple tissues, such as the submucosa and the musculature, which is composed of smooth muscle cells (SMCs) and interstitial cells of Cajal $[1,2]$. The process of digestive mesenchyme development into SMCs is commonly decomposed into two major steps [3]. Mesenchymal progenitor cells first

\footnotetext{
* Correspondence: sandrine.faure@inserm.fr

${ }^{\dagger}$ Equal contributors

PhyMedExp, INSERM U1046, CNRS UMR 9214, University of Montpellier, 34295 Montpellier, France
}

enter a determination program (that we will refer to as SMC determination), mainly characterized by the early expression of alpha smooth muscle actin ( $\alpha \mathrm{SMA})$. Later during development, determined SMCs enter a more differentiated state (that we will refer to as SMC differentiation), mainly characterized by the expression of proteins involved in smooth muscle contractility, such as CALPONIN and CALDESMON.

Unlike many other mature cell types in the adult body, such as skeletal muscle cells, SMCs do not terminally differentiate but instead harbour a remarkable capacity to dedifferentiate. Indeed, SMCs have the unique ability to switch between a differentiated, quiescent contractile state and a highly proliferative and migratory phenotype in response to internal or external cues $[1,4]$. SMC plasticity plays crucial roles in maintaining muscular tissue homeostasis during perinatal development and postnatal 
stages. In humans, the disruption of this balance is a major underlying cause of disease $[4,5]$. Because tissue plasticity involves the reactivation of developmental processes, developmental studies of the process regulating the differentiation of mesenchymal progenitors into SMCs have proven to be useful in identifying the molecular mechanisms involved in the regulation of digestive musculature plasticity during normal development and in pathological conditions [6, 7].

Using a microarray approach to identify candidate genes in stomach mesenchyme development [8], an approach that had already enabled our group to characterize the RNA-binding protein RBPMS2 as a regulator of SMC differentiation and plasticity [6,9], we screened for genes that demonstrated higher expression at the earliest stages of stomach development. This allowed us to identify Limb Expression 1 (LIX1), a gene coding for a 281-amino acid protein. Although predictive in silico studies have shown that LIX1 has a double-stranded RNA binding domain, suggesting that it could be involved in RNA processing [10], no cellular function of LIX1 has yet been described. Chicken (Gallus gallus) LIX1, first identified in a gene expression screen to identify new markers of limb development, was shown to be expressed in the anterior and posterior intestinal portals, the early structures that invaginate to give rise to the primary intestinal tube [11]. Moreover, the arthropod homolog of LIX1, lowfat, has been characterized, through its interaction with the atypical cadherins fat and dachsous, as a component of the Hippo pathway $[10,12]$. The Hippo pathway has been at the centre of many studies regarding its role in maintaining tissue homeostasis through the regulation of the balance between cell proliferation and differentiation. The key downstream regulator of the Hippo pathway is Yes-Associated Protein (YAP1), a transcriptional co-activator that mainly interacts with transcription factors of the TEAD family, which are essential in mediating YAP-dependent gene expression [13-15]. Indeed, the Hippo core cascade of kinases is activated when proper cell density and organ size are reached, leading, in humans, to the inhibitory phosphorylation of YAP1 on Serine-127 [16, 17]. This leads to decreased transcription of YAP1 mitogenic targets, resulting in a decrease in cell proliferation and entry into a more differentiated state [17]. Although LIX1 has recently been shown to stimulate progenitor proliferation during skeletal muscle regeneration in mouse [18], there is no evidence to date to support a role for LIX1 in regulating the activity of YAP1 in vertebrates.

In the present study, we investigated the roles of LIX1 and YAP1 during digestive SMC development. We show that LIX1 is a novel and, thus far, unique marker of stomach mesenchymal progenitors and that its expression is strong and highly dynamic during development.
We demonstrate that LIX1 stimulates the expression of YAP1 transcripts and YAP1 activity and that both LIX1 and YAP1 are key regulators of the development of stomach mesenchymal progenitors. Finally, we show that YAP1 activity is required for the regulation of the proliferation and differentiation of the stomach mesenchyme.

\section{Results}

\section{LIX1 defines stomach mesenchymal progenitors}

We previously screened for genes that demonstrated higher expression at the earliest stages of stomach development [8] and found LIX1 to be among them. Real-time quantitative PCR (RT-qPCR) analyses on stomach extracts confirmed the dynamic and transitory nature of LIX1 expression during stomach development (Additional file 1: Figure S1A). While high levels of LIX1 transcripts were detected at embryonic day 4 (E4), levels of LIX1 transcripts quickly decreased with the onset of SMC determination (as visualized through the expression of $\alpha S M A$ and SM22), to finally barely detectable levels at E7, when SMC differentiation occurred (as shown by the high level of CALPONIN and CALDESMON expression; Additional file 1: Figure S1A). In parallel, we monitored the levels of $B A R X 1$, a marker of stomach mesenchyme [19], as well as SRF and its co-activator MYOCARDIN, which control SMC differentiation [20,21], and found that, while the onset of MYOCARDIN expression occurs at E5.5, the stage of SMC determination, SRF and BARX1 were detected throughout all examined stages. These results suggest that LIX1 is an early marker of stomach development. We further studied the precise expression pattern of LIXI in the developing GI tract by in situ hybridization analysis (Additional file 1: Figure S1B). Strong LIX1 expression was detected at E4 throughout the stomach mesenchyme and levels quickly decreased from E5 onwards (Fig. 1a, b). LIX1 transcripts were mainly detected in the pylorus at E5 and in the most posterior part of the stomach at E6 (Fig. 1a, b). When comparing the dynamics of LIX1 expression in the developing stomach with the kinetics of $\alpha \mathrm{SMA}$, the early marker of SMC determination in adjacent stomach sections, we observed that their expression domains appeared mutually exclusive (Fig. 1b). While LIX1 expression was high in stomach mesenchymal progenitors, it progressively decreased with the onset of SMC determination, thus identifying LIX1 as a novel and unique stomach marker, restricted to mesenchymal progenitors (Fig. 1c).

\section{LIX1 silencing impairs mesenchyme determination and decreases YAP1 activity}

The complementarity between LIX1 and $\alpha$ SMA expression prompted us to investigate whether $L I X 1$ is required 

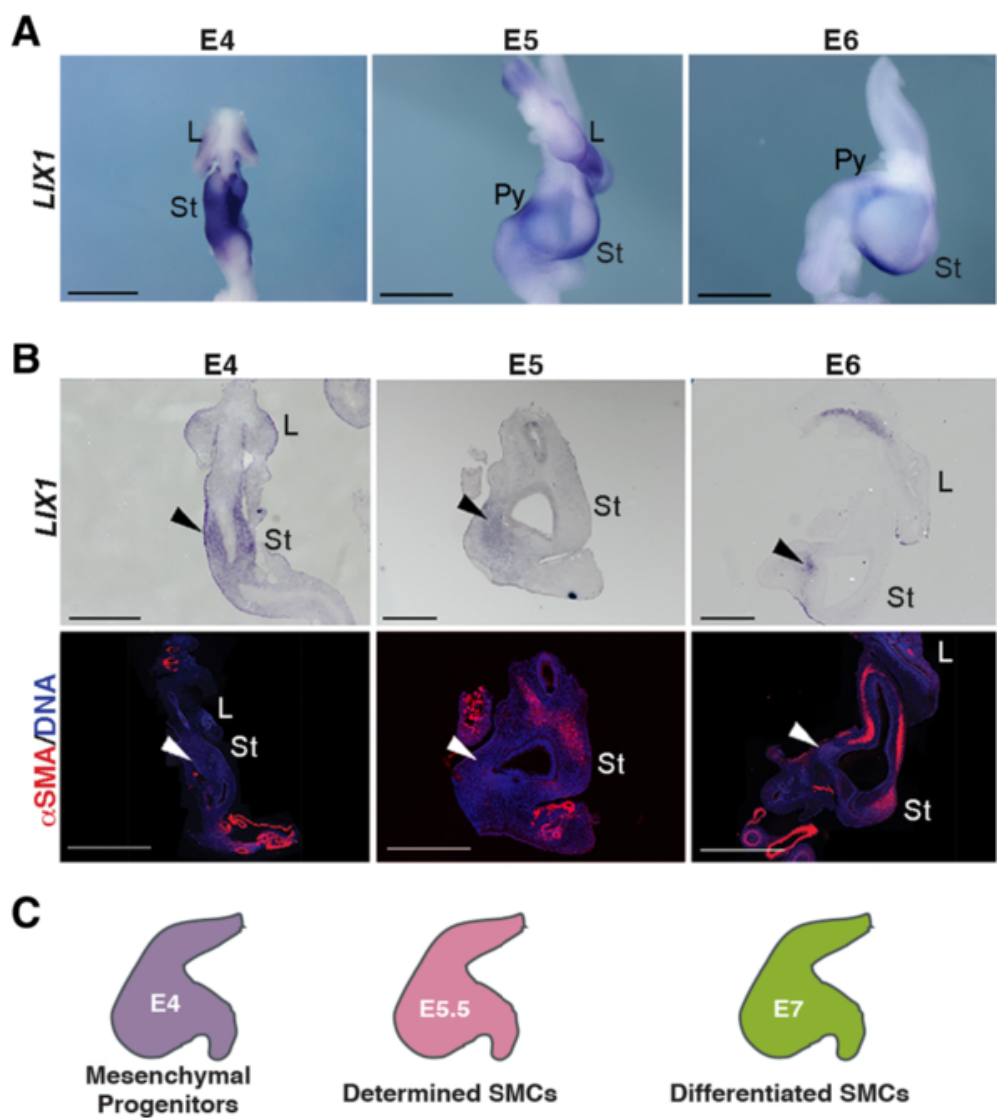

Differentiated SMCs

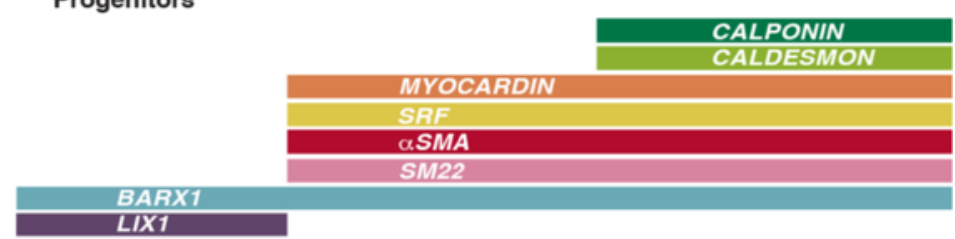

Fig. 1 Transient expression pattern of $L I X 1$ in the developing chick stomach. a $L I X 1$ whole-mount in situ hybridization of embryonic day 4 (E4) to E6 stomachs. Scale bars, $1 \mathrm{~mm}$. b Serial longitudinal sections of E4 to E6 stomachs analysed by in situ hybridization using the LIX1 riboprobe and by immunofluorescence with anti-aSMA antibodies. Nuclei are visualized with Hoechst. Black arrowheads show the mesenchymal expression of $L I X 1$ at these stages. White arrowheads show the absence of aSMA in the LIX1-expressing domains. Scale bars, $500 \mu \mathrm{m}$. c Cartoon illustrating the steps of stomach mesenchyme development and summarizing Fig. 1a, b and Additional file 1: Figure S1A. L, Lung; St, Stomach; Pyl, Pylorus

for the process of stomach SMC determination. This was accomplished using the avian replication-competent retroviral (RCAS) transgenesis method that allows in vivo gain- or loss-of-function approaches of specific genes in the stomach mesenchyme (Additional file 2: Figure S2A) $[6,8,19,22]$. We first performed LIX1 loss-offunction experiments using RCAS(A)-ShLIX1 (shorthairpin RNA directed against $L I X 1$ ) retroviruses. When injected into the presumptive domain of the developing stomach, RCAS(A)-ShLIX1 retroviruses led to a specific decrease in endogenous LIX1 expression, demonstrated by in situ hybridization and RT-qPCR analyses (Fig. 2a, c). In situ hybridization analysis revealed a decrease in the expression of the SMC determination marker SM22 in E6.5 ShLIX1-expressing stomachs compared to controls
(Fig. 2b) upon LIX1 silencing. This was confirmed by RT-qPCR analysis (Fig. 2c). In contrast, injection of unrelated RCAS(A)-ShRNA retroviruses, which do not target LIX1, had no effect on $\alpha S M A$ expression (Additional file 3: Figure S3A). Moreover, when RCAS(A)-ShLIX1 retroviruses were co-injected with RCAS(B)-hLIX1 retroviruses, which induce the expression of the human LIX1 protein insensitive to the chick-specific RCAS(A)-ShLIX1 retroviruses, normal expression of $\alpha S M A$ was restored, demonstrating the specificity of the ShLIX1 construct for LIX1 (Additional file 3: Figure S3B). Levels of BARX1 transcripts were comparable in ShLIX1-expressing stomachs compared to controls, indicating that the patterning of the stomach was unaffected by LIX1 silencing (Fig. 2c). We also observed a decrease in MYOCARDIN 

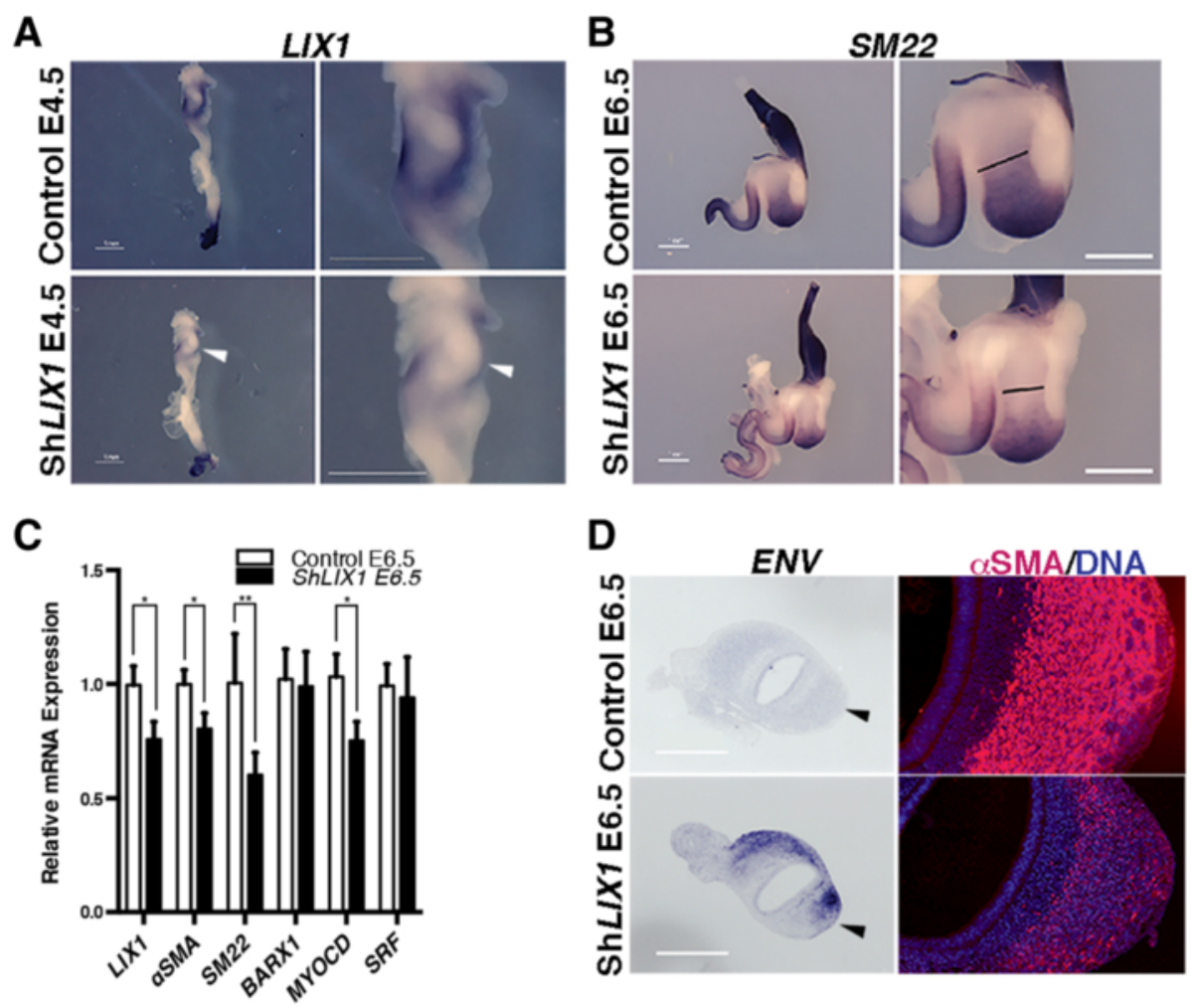

E
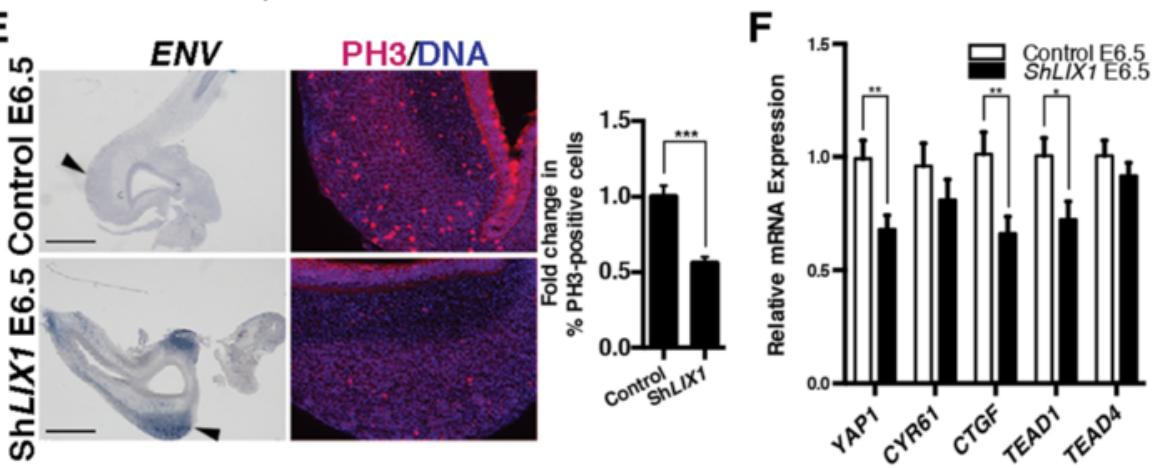

Fig. $2 L I X 1$ is required for mesenchymal progenitor proliferation and smooth muscle cell determination in the developing stomach. a $L I X 1$ whole-mount in situ hybridization of E4.5 gastrointestinal tracts. Scale bars, $1 \mathrm{~mm}$. White arrowheads show the down-regulation of LIXI expression in ShLIX1-expressing stomachs. b SM22 whole-mount in situ hybridization of gastrointestinal tracts. Black bars locate the change in the SM22-expression domain. c RT-qPCR analysis of relative mRNA expression. Data were normalized to GAPDH expression. Normalized expression levels were converted to fold changes. Values are presented as the mean \pm standard error of the mean (SEM) of $n=12$ controls vs. $n=10$ ShLIXIexpressing stomachs. ${ }^{*} P<0.05$; ${ }^{* *} P<0.01$ by one-tailed ( $L I X 1$, aSMA and SM22) or two-tailed (BARX1, MYOCD and SRF) Mann-Whitney tests. d Serial transverse sections of stomachs analysed either by in situ hybridization using the retroviral Envelop (ENV) riboprobe (scale bars, $500 \mu \mathrm{m}$ ) or by immunofluorescence with anti-aSMA antibodies. Nuclei were visualized with Hoechst. Black arrowheads in the ENV panels indicate the area that is imaged at high power in the aSMA panels. e Serial transverse sections of stomachs analysed either by in situ hybridization using the ENV riboprobe (scale bars, $500 \mu \mathrm{m}$ ) or by immunofluorescence using anti-PH3 antibodies. Nuclei are visualized with Hoechst. Black arrowheads in the ENV panels indicate the area imaged at high power in the PH3 panels. Graph represents the quantification of PH3-positive cells. Normalized expression levels were converted to fold changes. Values are presented as the mean \pm SEM of $n=12$ control vs. $n=10$ ShLIX1-expressing stomachs. ${ }^{* * *} P<0.001$ by two-tailed Mann-Whitney test. $\mathbf{f R T - q P C R}$ analysis of relative mRNA expression in control and ShLIX1-expressing stomachs. Data were normalized to GAPDH expression. Normalized expression levels were converted to fold changes. Values are presented as the mean \pm SEM of $n=12$ controls vs. $n=10$ ShLIX1-expressing stomachs. ${ }^{*} P<0.05 ;{ }^{* *} P<0.01$ by two-tailed Mann-Whitney tests

expression, while levels of $S R F$ transcripts were not significantly affected in E6.5 ShLIX1-expressing stomachs compared to controls (Fig. 2c). LIX1 silencing induced a smaller determined-SMC territory, as demonstrated by in situ hybridization (Fig. 2b) and immunostaining analyses on ShLIX1-expressing stomach sections compared to controls (Fig. 2d; Additional file 4: Table S1). The diminished expression of SMC determination markers was associated 
with a $40 \%$ decrease in the rate of cell proliferation in ShLIX1-expressing stomach sections compared to controls, as demonstrated by immunostaining analysis for phosphorylated histone 3-Ser10 (PH3; Fig. 2e), a standard marker of the G2/M transition [6]. These results are in line with a role for LIX1 in regulating cell proliferation, as previously shown in studies on cricket (Gryllus bimaculatus) and mouse that identified homologs of LIX1 as positive regulators of cell proliferation $[10,18]$. Lowfat, the arthropod homolog of LIX1, has been characterized, through its interaction with the atypical cadherins fat and dachsous, as a component of the Hippo pathway [10, 12]. As the key downstream regulator of the Hippo pathway is the pro-proliferative gene YAP1, we next investigated whether LIX1 regulates the expression of YAP1 during this process. In situ hybridization and RT-qPCR analyses revealed that endogenous transcripts of YAP1 and its transcriptional targets CTGF and CYR61, known to stimulate cell proliferation $[15,23]$, are abundant during early development of the stomach (E4-5.5; Additional file 5: Figure $\mathrm{S} 4 \mathrm{~A}, \mathrm{~B})$. At this stage, their expression is detectable in both the mesenchymal and epithelial layers of the stomach, as demonstrated by RT-qPCR analyses on layer-dissociated stomach extracts (Additional file 5: Figure S4C). RT-qPCR analysis showed a reduction in the level of YAP1 and its transcriptional targets $C T G F$ and CYR61 in ShLIX1-expressing stomachs compared to controls (Fig. 2f). Although expression data were significant for CTGF, but not for CYR61, the results for both transcripts were consistent. We attribute the lack of significance for the second transcript to low statistical power rather than to absence of an effect. These results indicate that YAP1 activity was decreased in ShLIX1-expressing stomachs compared to controls. Moreover, LIX1 silencing also induced a decrease in the expression of the TEAD transcription factor TEAD1 (Fig. 2f). Taken together, our results show that, when LIX1 expression was silenced in the developing stomach, SMC determination was hindered. This was associated with a decrease in cell proliferation and a decrease in YAP1 transcript levels and YAP1 activity in the developing mesenchyme. Our finding highlights the requirement of LIX1 expression in the stomach mesenchymal progenitors to establish normal proliferation rates and allow proper SMC determination.

LIX1 misexpression expands the determined SMC domain and stimulates cell proliferation and YAP1 activity

We next induced a misexpression of LIX1 in the stomach mesenchyme using RCAS(B)-LIX1 retroviruses (Additional file 2: Figure S2A). This treatment did not drastically affect GI morphogenesis, as the morphology of LIX1-misexpressing stomachs resembled that of control embryos (Additional file 2: Figure S2B). We first observed a premature expression of SMC determination marker SM22 as early as E4.5 in LIX1-misexpressing stomachs, whereas SMC determination had not yet taken place in controls, suggesting that LIXI misexpression facilitated SMC determination (Fig. 3a, white arrowhead). As a result, we observed at E6 that LIX1-misexpressing stomachs harboured an expanded determined SMC territory at the expense of the adjacent domains, mainly the intermuscular tendons and the submucosa. This was demonstrated both by whole-mount in situ hybridization, which showed a larger expression domain of determined SMC markers SM22 and BAPX1 [24] in LIX1-misexpressing stomachs compared to controls (Fig. 3b), and by $\alpha$ SMA immunostaining on sections, showing that sustained LIX1 expression led to a decrease in the size of the submucosa (Fig. 3c, compare white bars). Accordingly, analysis of the enteric nervous system network using in situ hybridization of SOX10 transcripts revealed that enteric nervous system precursors, which normally colonize the SMC domain specifically [8], had migrated into the adjacent tendon territory, further indicating an expanded SMC domain in LIX1-misexpressing stomachs compared to controls (Fig. 3b, white arrowhead). Further analysis by RT-qPCR confirmed that, compared to control stomachs, LIX1-misexpressing stomachs harboured higher levels of SMC determination marker $\alpha S M A$ and BARX1 transcripts at E6, whereas MYOCARDIN and SRF levels were not significantly affected (Fig. 3d). Taken together, our in vivo results indicate that LIX1 is not only necessary for correct SMC determination, but that it also acts in favour of the process. These changes are associated with an increase in the rate of cell proliferation, as demonstrated by immunostaining analysis for $\mathrm{PH} 3$, and consequently to an increase in mesenchymal cell density in E6 LIX1-misexpressing stomachs compared to controls (Fig. 3e). The rate of cell death, however, was comparable in both conditions, as demonstrated by immunostaining analysis of cleaved CASPASE-3 (Additional file 6: Figure S5). Taking into account the positive effect of LIX1 on SMC proliferation and our previous results demonstrating that LIX1 silencing impaired YAP1 expression and activity, we suspected that LIX1 overexpression would stimulate the expression of genes in the YAP1 pathway. In fact, RT-qPCR analysis indicated a significant increase in the expression of YAP1, CTGF and TEAD1, and a slight increase in the expression of CYR61 and TEAD4 in LIX1misexpressing stomachs compared to controls (Fig. 3f).

The differences in YAP1 expression and activity observed in LIX1-misexpressing stomachs could be linked to the changes in the identity of the tissue associated with aberrant LIX1 expression, or could be due to a role of YAP1 as a key relay in the establishment of the LIX1 phenotype. We thus performed YAP1 gain-of-function 


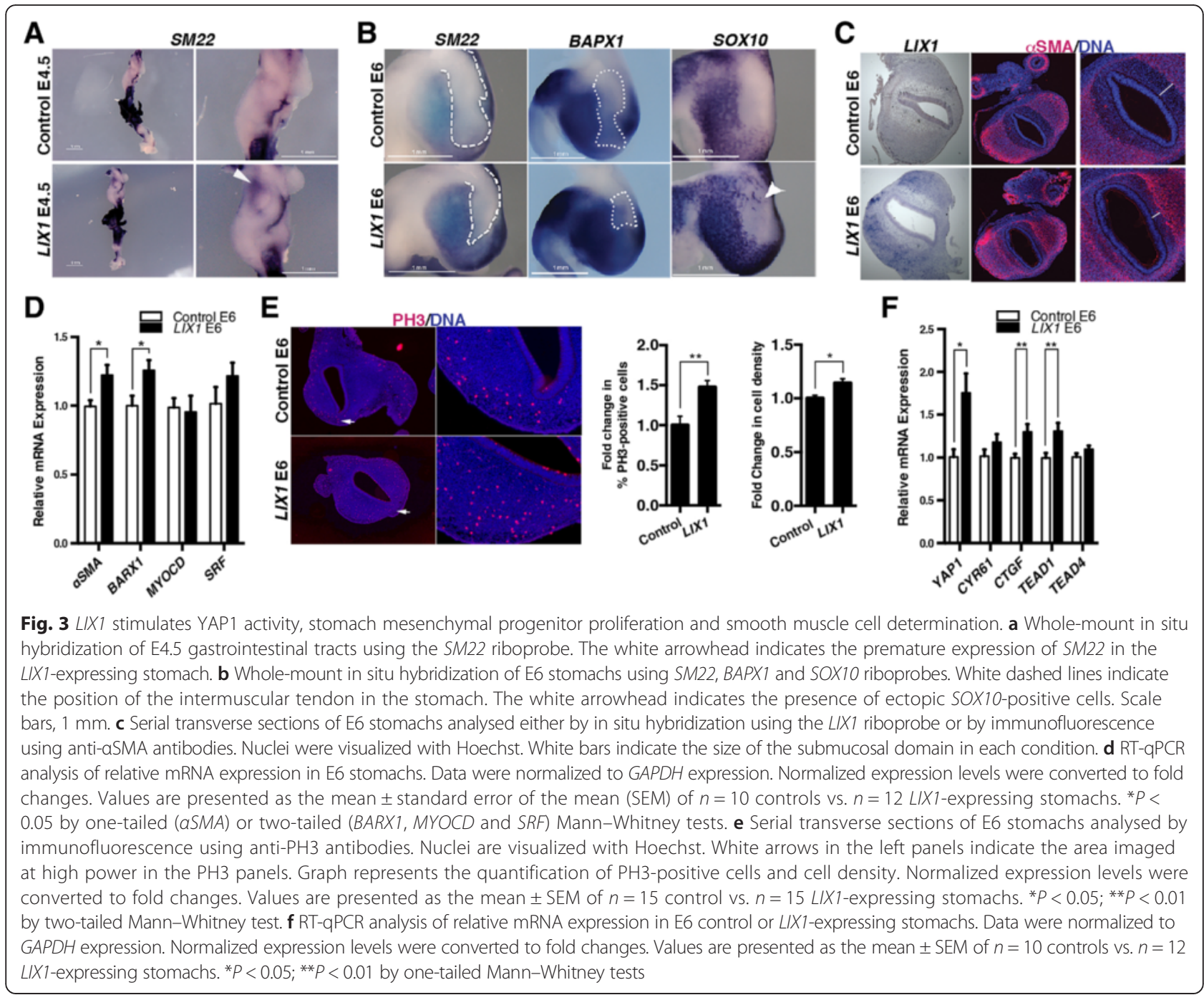

experiments in the developing stomach using RCAS(B)YAP1 retroviruses. We observed an expanded SM22positive determined SMC domain in YAP1-misexpressing stomachs compared to control stomachs (Fig. 4a). RTqPCR analysis indicated that YAP1 misexpression did not affect the endogenous expression of LIX1 (data not shown) and confirmed an increase in the transcript levels of the SMC determination markers $\alpha S M A$ and MYOCARDIN at E6 (Fig. 4b). Levels of BARX1, SRF, $T E A D 1$ and TEAD4 were not significantly affected in YAP1-misexpressing stomachs compared to control stomachs (Fig. 4b, d). Moreover, changes in expression of SMC determination markers were associated with an increase in cell proliferation, as demonstrated by immunostaining analysis for PH3 (Fig. 4c). Our results thus demonstrate that LIX1 stimulates the endogenous level of YAP1 transcripts and YAP1 activity and that sustained YAP1 activity phenocopies LIX1 misexpression regarding stomach mesenchyme determination.
Furthermore, when RCAS(A)-ShLIX1 retroviruses were co-injected with RCAS(B)-YAP1 retroviruses, the expression of LIX1 was not rescued (Fig. 4e). However, the restored YAP1 activity (monitored through the expression of CYR61 and CTGF transcripts) rescued the expression of $\alpha S M A$ (Fig. 4e). Altogether, these data demonstrate that YAP1 is a key relay in the establishment of the LIX1 phenotype.

\section{Endogenous LIX1 expression is regulated by the FGF pathway during SMC determination}

Collectively, our in vivo loss- and gain-of-function experiments demonstrate that LIX1 expression must be finely regulated in the stomach mesenchyme to control the pool of progenitors required for correct SMC determination, presumably through the regulation of YAP1 activity. It has been shown that aberrant activation of the FGF pathway has a negative impact on stomach SMC determination [8]. As we report that LIX1 silencing 

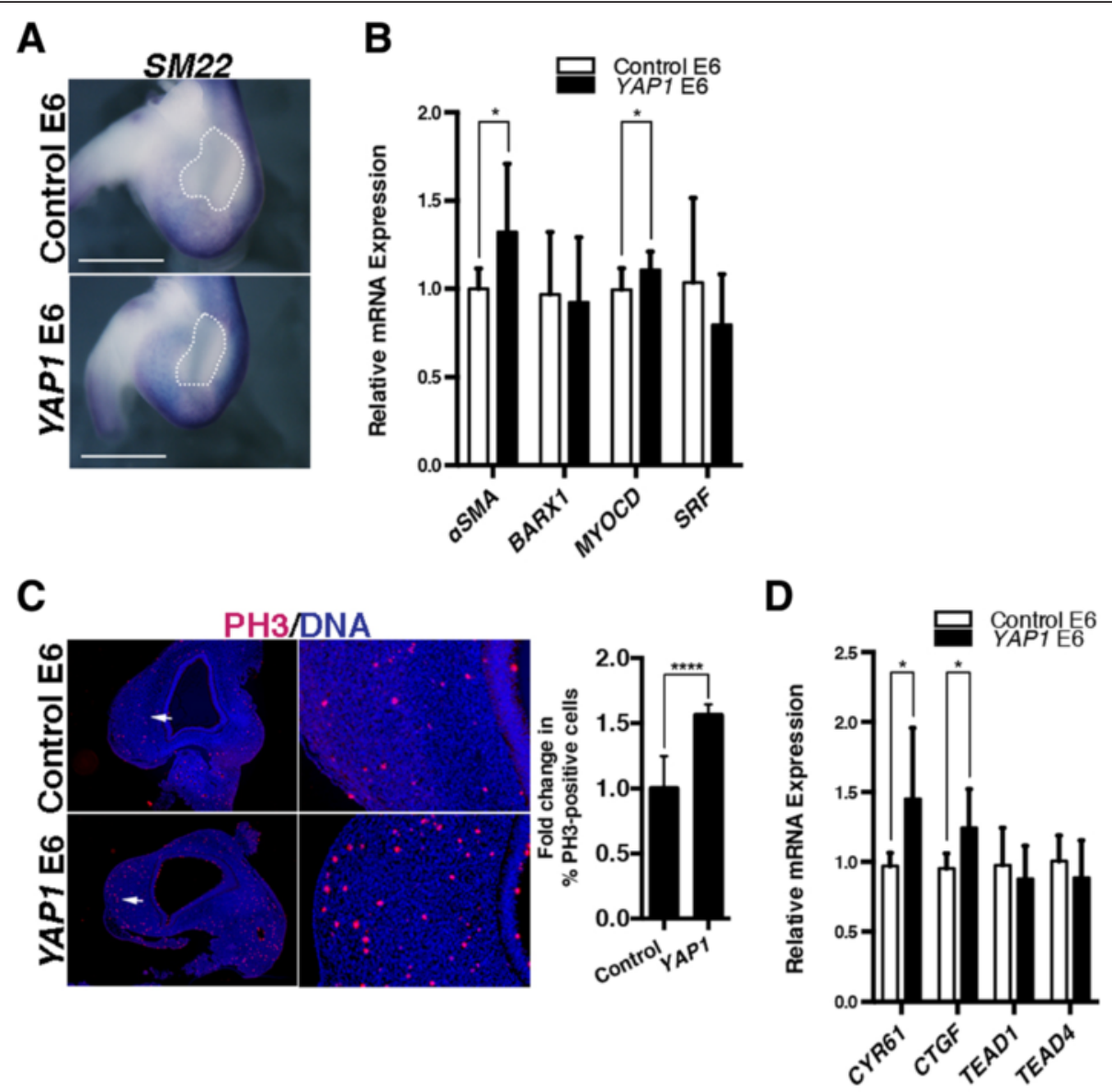

E

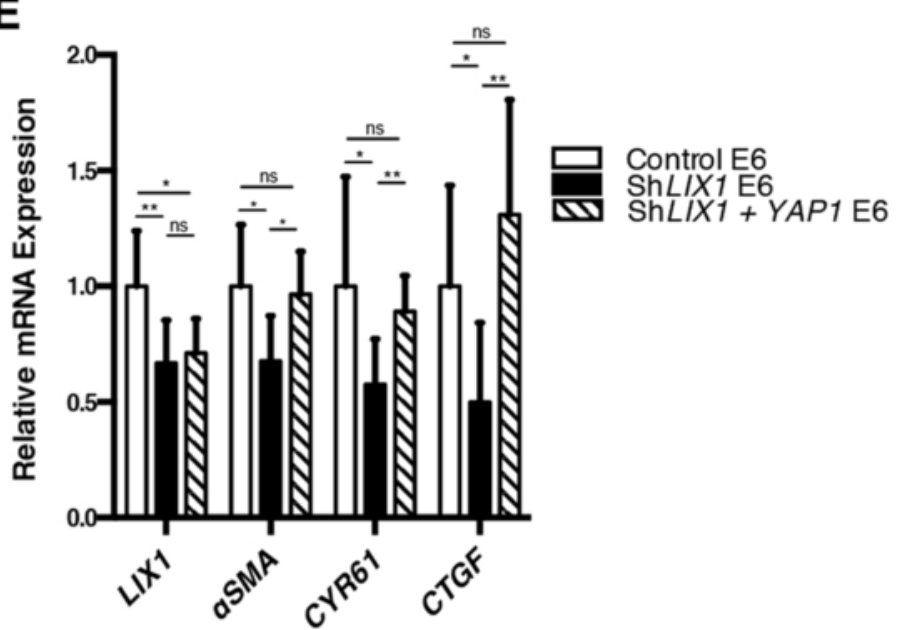

Fig. 4 (See legend on next page.) 
(See figure on previous page.)

Fig. 4 YAP1 stimulates stomach mesenchymal progenitor proliferation and smooth muscle cell determination. a Whole-mount in situ hybridization of E6 stomachs using the SM22 riboprobe. White dashed lines indicate the position of the intermuscular tendon in the stomach. Scale bars, $1 \mathrm{~mm}$. b RT-qPCR analysis of relative mRNA expression in E6 stomachs. Data were normalized to GAPDH expression. Normalized expression levels were converted to fold changes. Values are presented as the mean \pm standard derivation (SD) of $n=8$ controls vs. $n=6$ YAP1-expressing stomachs. ${ }^{*} P<0.05$ by one-tailed Mann-Whitney tests. $\mathbf{c}$ Serial transverse sections of E6 stomachs analysed by immunofluorescence using anti-PH3 antibodies. Nuclei are visualized with Hoechst. White arrows in the left panels indicate the area imaged at high power in right panels. Graph represents the quantification of PH3-positive cells. Normalized expression levels were converted to fold changes. Values are presented as the mean \pm standard error of the mean of $n=10$ controls vs. $n=10$ YAP1-expressing stomachs. ${ }^{* * * *} P<0.0001$ by two-tailed Mann-Whitney test. $\mathbf{d}$ RT-qPCR analysis of relative mRNA expression in E6 stomachs. Data were normalized to GAPDH expression. Normalized expression levels were converted to fold changes. Values are presented as the mean \pm SD of $n=8$ controls vs. $n=6$ YAP1-expressing stomachs. ${ }^{*} P<0.05$ by one-tailed Mann-Whitney test. e RT-qPCR analysis of relative mRNA expression in E6 stomachs. Data were normalized to GAPDH expression. Normalized expression levels were converted to fold changes. Values are presented as the mean \pm SD of $n=8$ controls vs. $n=8$ ShLIXI vs. $n=6$ ShLIXI + YAP1-expressing stomachs. ${ }^{*} P<0.05$; ${ }^{* *} P<0.01$ by one-tailed Mann-Whitney test. ns, not significant

impaired SMC determination, we next investigated whether the expression of LIX1 was under the control of the FGF signalling pathway. To address this question, we activated the FGF signalling pathway by misexpressing FGF8 in the stomach mesenchyme using RCAS(A)-FGF8 retroviruses and confirmed by RT-qPCR analysis that mesenchyme determination was hindered, as demonstrated by lower levels of $\alpha S M A$ and SM22 transcripts in FGF8-misexpressing stomachs compared to controls (Fig. 5b). The upregulation of FGF activity was associated with a strong reduction in LIX1 transcript levels compared to control stomachs, which was monitored by in situ hybridization (Fig. 5a) and confirmed by RT-qPCR analysis (Fig. 5b), and this was associated with a decrease in the levels of YAP1 transcripts (Fig. 5b). These results suggest that sustained FGF activity during SMC determination phenocopies LIX1 loss-of-function. Conversely, when using RCAS(B)$\mathrm{sFGFR} 2 b$ retroviruses, which produce a secreted form of FGFR2b $[8,25]$, we found that inhibition of FGF pathway activity induced an increase in LIX1 levels at E6.5 compared to control stomachs (Fig. 5c, white arrows). Taken together, these results suggest that the FGF pathway regulates the endogenous expression of LIX1 and thereby maintains the proper levels necessary to ensure correct stomach mesenchyme determination.

\section{Sustained LIX1 expression decreases YAP1 activity and hinders SMC differentiation}

To further understand the role of LIX1 in the development of the stomach mesenchyme, we next analysed the consequences of LIX1 misexpression on SMC differentiation, the later step of SMC development. We found a reduction in the level of CALPONIN protein at E7 in LIX1-misexpressing stomachs, indicating that SMC differentiation was impaired (Fig. 6a). A strong reduction in CALPONIN transcript levels was also observed (Fig. 6b). Additionally, we observed a decrease
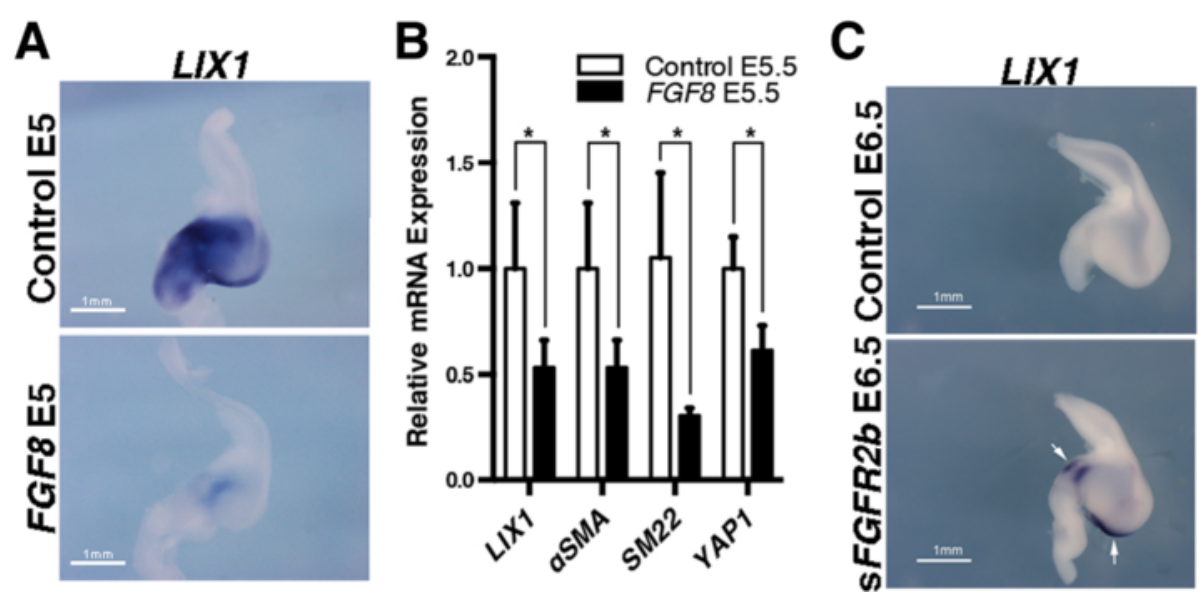

Fig. 5 Endogenous $L I X 1$ expression is regulated by the FGF pathway during smooth muscle cell determination. a $L I X 1$ whole-mount in situ hybridization of E5 control GFP- and FGF8-expressing stomachs. b RT-qPCR analysis of relative mRNA expression in E5 stomachs. Data were normalized to GAPDH expression. Normalized expression levels were converted to fold changes. Values are presented as the mean \pm standard deviation of $n=4$ controls vs. $n=3$ FGF8-expressing stomachs. ${ }^{*} P<0.05$ by one-tailed Mann-Whitney test. Raw data are shown in Additional file 12. c LIX1 whole-mount in situ hybridization of E6.5 control GFP- and sFGFR2b-expressing stomachs. Scale bars, $1 \mathrm{~mm}$. White arrows show the domain of the sFGFR2b-expressing stomach in which the expression of LIXI is sustained 

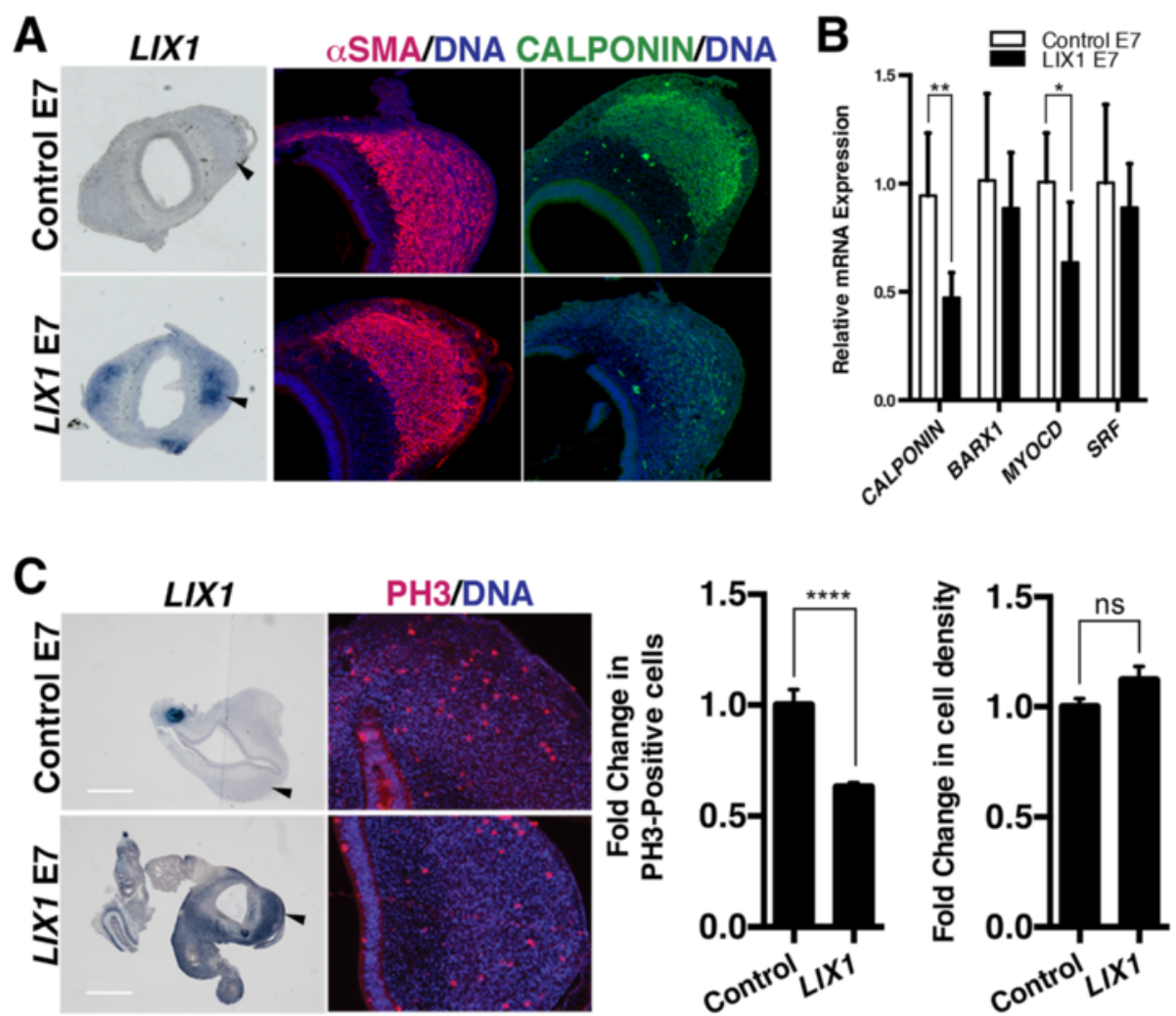

D

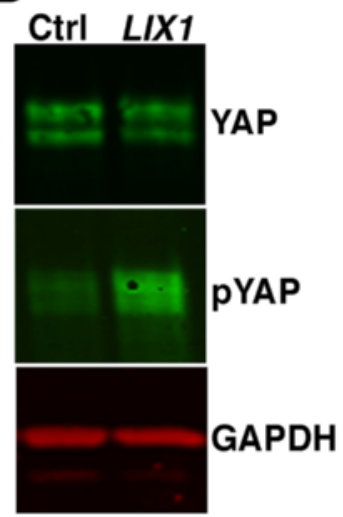

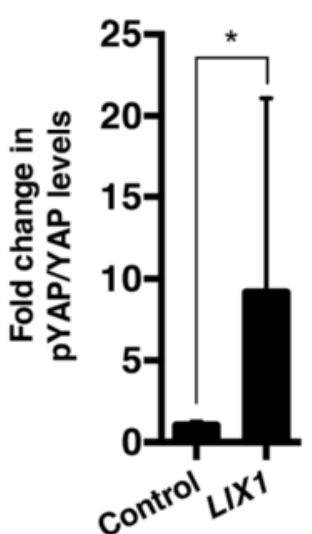

$\mathbf{E}$

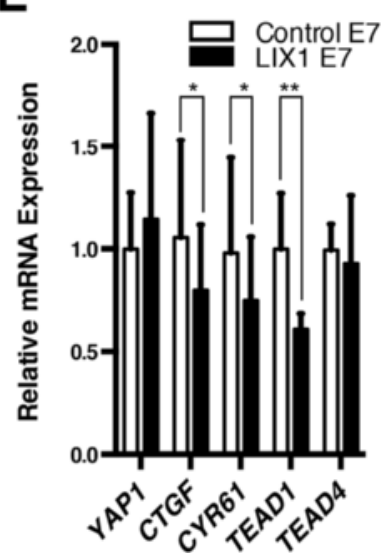

Fig. 6 Sustained LIX1 expression decreases YAP1 activity and hinders smooth muscle cell differentiation. a Serial transverse sections of E7 stomachs analysed either by in situ hybridization using the LIXI riboprobe or by immunofluorescence with anti-aSMA and anti-CALPONIN antibodies. Nuclei were visualized with Hoechst. Black arrowheads in the LIXI panels indicate the area imaged at high power in the aSMA and CALPONIN panels. b RT-qPCR analysis of relative mRNA expression in E7 stomachs. Data were normalized to GAPDH expression. Values are presented as the mean \pm standard deviation (SD) of $n=7$ controls vs. $n=6$ LIX1-expressing stomachs. ${ }^{*} P<0.05$; ${ }^{* *} P<0.01$ by one-tailed Mann-Whitney test. c Serial transverse sections of E7 stomachs analysed either by in situ hybridization using the LIXI riboprobe or by immunofluorescence with anti-PH3 antibodies. Black arrowheads in the LIX1 panels indicate the area imaged at high power in the PH3 panels. Nuclei were visualized with Hoechst. Scale bars, $500 \mu \mathrm{m}$. Graphs represent the quantification of PH3-positive cells and cell density. Normalized expression levels were converted to fold changes. Values are presented as the mean \pm standard error of the mean of $n=16$ controls vs. $n=16$ LIX1-expressing stomachs. ${ }^{* * *} P<0.0001$ by two-tailed Mann-Whitney tests. ns, not significant. $\mathbf{d}$ Western blot analysis of YAP and phospho-YAP (72 kDa) protein levels. Equal loading was verified by GAPDH expression (37 kDa). Graph represents the quantification of western blot data. Values are presented as the mean \pm SD of $n=4$ controls vs. $n=4$ LIX1-expressing stomachs. ${ }^{*} P<0.05$ by one-tailed Mann-Whitney test. Raw data are shown in Additional file 12. e RT-qPCR analysis of relative mRNA expression in E7 stomachs. Data were normalized to GAPDH expression. Normalized expression levels were converted to fold changes. Values are presented as the mean \pm SD of $n=7$ controls vs. $n=6$ LIX1-expressing stomachs. ${ }^{*} P<0.05 ;{ }^{*} P<0.01$ by one-tailed Mann-Whitney tests 
in the expression of MYOCARDIN, while levels of BARX1 and SRF transcripts were not significantly affected (Fig. 6b). The decrease in SMC differentiation markers in LIX1-misexpressing stomachs was also observed later in development at E8.5, suggesting that the reduced level of differentiation markers did not simply reflect a delay in stomach SMC development (Additional file 7: Figure S6). We found that YAP1 misexpression also hindered CALPONIN expression, as demonstrated by immunostaining on stomach sections (Additional file 8: Figure S7A). This suggests that, while LIX1 misexpression and YAP1 stimulation had a positive impact on SMC determination, they hindered SMC differentiation. Surprisingly, we found that, when LIX1 expression was sustained in the developing stomach, the downregulation in the expression of SMC differentiation markers was associated with a lower rate of proliferation (Fig. 6c). Indeed, mesenchymal cell density was comparable in LIX1-misexpressing stomach compared to controls (Fig. 6c). It has been shown that the Hippo pathway acts as a sensor of cell density $[16,17]$, thus mediating the relationship between cell proliferation and cell contact inhibition of proliferation. As cell density becomes higher, the Hippo pathway is activated, resulting in an inhibitory phosphorylation of YAP1 and thus a decrease in cell proliferation [26]. Interestingly, we observed a decrease in YAP1 activity in YAP1-misexpressing stomachs at E7 by western blot analysis monitored through an increase of the inactive phosphorylated form of YAP1 compared to controls (Additional file 8: Figure S7B). The decrease in YAP1 activity was confirmed by RT-qPCR analysis, which showed lower transcript levels of CTGF in YAP1-misexpressing stomachs at E7 compared to controls (Additional file 8: Figure S7C). These results indicate that, while YAP1 misexpression in the stomach stimulated YAP1 transcriptional activity at determination stages, a decrease in YAP1 activity was observed later on at differentiation stages. One possible explanation is that sustained LIXI expression led to a decrease in YAP1 activity consecutive to cell contact inhibition of proliferation, as a consequence of the early stimulation of mesenchymal progenitor proliferation, and this could be inhibitory for SMC differentiation. In line with this hypothesis, western blot analysis revealed an increase of the inactive phosphorylated form of YAP1 compared to controls (Fig. 6d). The decrease in YAP1 activity in LIX1-misexpressing stomachs at E7 was further confirmed by RT-qPCR analysis, which showed lower transcript levels of YAP1 targets CYR61 and CTGF in LIX1-misexpressing stomachs compared to controls (Fig. 6e). Additionally, we observed a decrease in TEAD1 transcript levels in LIX1-misexpressing stomachs compared to controls (Fig. 6e). These data indicate that Hippo signalling was activated as a result of sustained LIX1 expression at E7. Altogether, our results demonstrate that LIX1 has an early role in the process of stomach SMC determination, through the regulation of YAP1-induced mesenchymal progenitor proliferation. However, as stomach development proceeds, sustained LIX1 expression has a negative impact on further SMC differentiation and this is associated with a decrease in YAP1 activity.

\section{The ability of LIX1 to regulate cell proliferation is dependent on cell density}

These results prompted us to investigate the role of LIX1 in regulating both proliferation and contact inhibition of proliferation in heterologous cell cultures. DF-1 chicken fibroblasts were infected with empty RCAS(A) (control) or RCAS(B)-LIX1 retroviruses and cultured for 5 days to ensure homogeneous expression. Then, cells were seeded at low density (Fig. 7a). As expected according to our in vivo results demonstrating a positive effect of LIX1 overexpression on the expression of YAP1 (Fig. 3f), after 1 day in culture (day 1) LIX1-expressing cells demonstrated a higher expression of YAP1 transcript (Fig. 7b) and protein levels (Fig. 7c) compared to control cells. This greater expression was associated with higher transcript levels of YAP1 target genes CTGF and CYR61 (Fig. 7b) and an increase in cell proliferation (Fig. 7d). Interestingly, when LIX1-expressing cells were treated with verteporfin, an inhibitor of the YAP-TEAD interaction that abrogates YAP activity but not its expression [27, 28], levels of CTGF and CYR61 transcripts (Fig. 7e) and rates of proliferation (Fig. 7f) were comparable with those of control cells. Analysis of cell death in these cultures confirmed that this result was not due to a cytotoxic effect of verteporfin (Fig. $7 \mathrm{~g}$ ). These data demonstrate that, at low density, LIX1 regulates cell proliferation through modulation of YAP1 activity. After 3 days in culture, LIX1-expressing cells had grown faster than control cells (Fig. 7h, i). However, although YAP1 expression in LIX1-expressing cells remained higher than in controls (Fig. 7j, k), the levels of CTGF and CYR61 transcripts were similar to control levels. In addition, we observed an increase of the inactive phosphorylated form of YAP1 compared to controls in LIX1expressing cells (Fig. 7k), indicating that YAP1 activity was downregulated at day 3 compared to day 1 (compare Fig. 7b with Fig. 7j). These data suggest that, under the influence of LIX1, a compensatory response to growing cell density took place. Indeed, while LIX1 acts to promote cell proliferation at low cell density, its proproliferation activity is abolished when cells had grown, suggesting that its ability to regulate cell proliferation is dependent upon cell density. In line with this hypothesis, when cells were seeded at high density, levels of CTGF and CYR61 transcripts, YAP1 activity, and rates of proliferation 


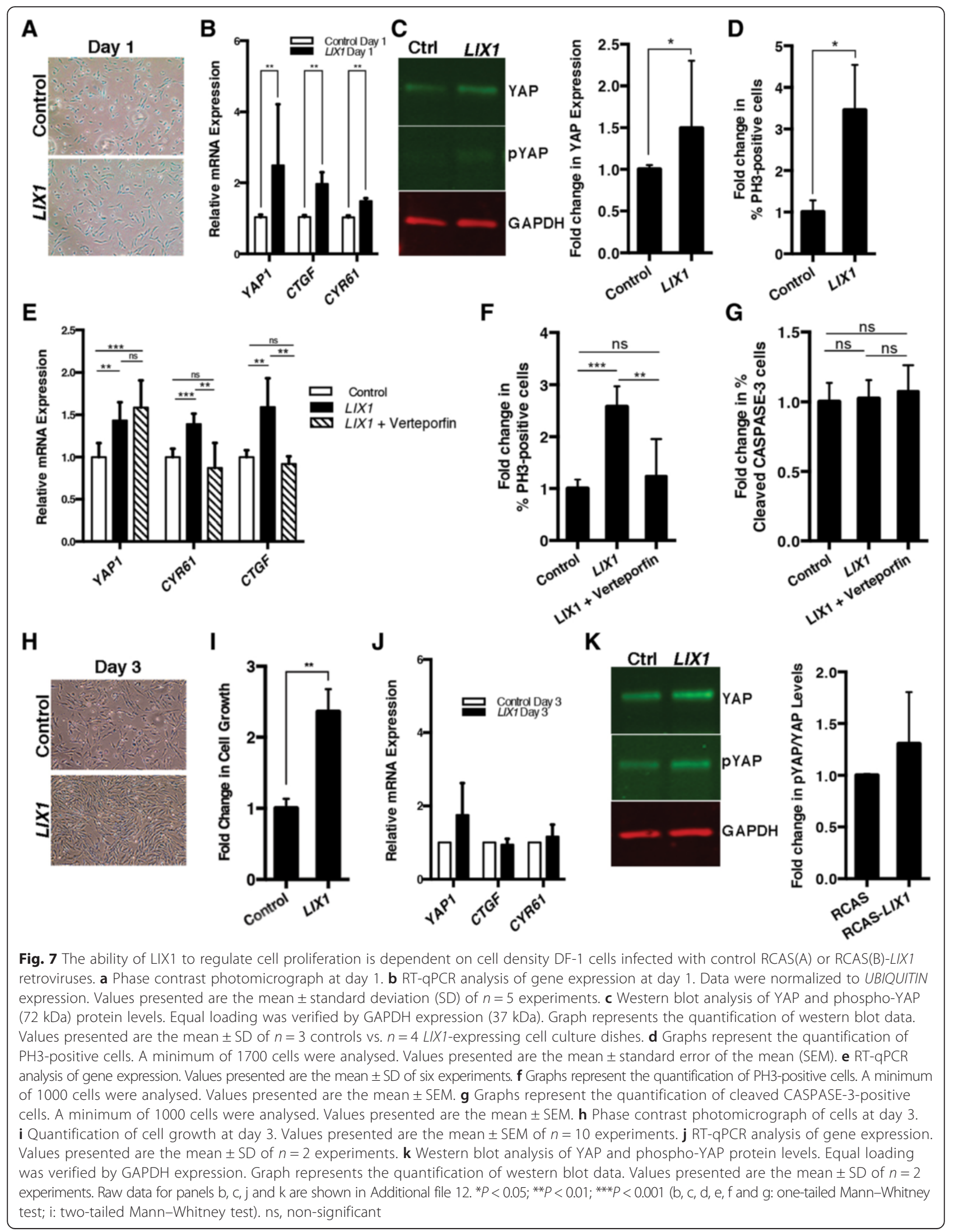


were comparable in controls and LIX1-expressing cells after 1 day in culture (Additional file 9: Figure S8). The overexpression of LIX1 in vitro thus recapitulates the effects we had observed under misexpression of LIX1 in vivo during stomach mesenchyme development, suggesting that LIX1 drives an increase in cell density that feeds back on the system to block the activity of YAP1 and further proliferation.

\section{Discussion}

Our study first identified LIX1 as a novel and thus far unique marker of stomach mesenchymal progenitors. To our knowledge, LIX1 is the first described gene to define the population of mesenchymal progenitors and to allow discrimination between undetermined and determined SMC states in the stomach. Collectively, our in vivo gain- and loss-of-function experiments clearly demonstrate that LIX1 is a key regulator of stomach mesenchyme development, by regulating both the determination and the differentiation of SMCs. Our study further demonstrates that YAP1 is a key relay of the function of LIX1 during these developmental processes.

We first identified LIX1 as an essential regulator of stomach mesenchyme determination. We thus suspect that the expression of $L I X 1$ must be tightly regulated in the developing mesenchyme to allow fine-tuning of the transcript levels and the state of activation of the proproliferative transcriptional coactivator YAP1, which in turn controls the rates of proliferation required for correct SMC determination. We further show that the FGF signalling pathway could be involved in the regulation of LIX1 expression at determination stages. Most studies published so far have identified some regulators of YAP1 at the level of its activity, through its phosphorylation, localisation and stability [29]. Our study identifies LIX1 as a new regulator of YAP1 mRNA levels, which is a novel finding. This could result from a regulation of the transcription of YAP1 mRNA or from a regulation of its stability. Expression data were not always statistically significant for TEAD4. However, results were consistent between TEAD1 and TEAD across all experiments. We thus attribute the lack of significance in some cases of effects on TEAD4 to low statistical power rather than to absence of an effect. These functional in vivo data suggest that LIX1 also regulates the expression of the TEAD transcription factors, which are essential in mediating YAP-dependent gene expression [15], indicating that LIX1 is an upstream regulator of YAP signalling. Further investigations will allow us to understand by which mechanisms LIX1 regulates the level of YAP1 and TEAD transcripts. Interestingly, in silico studies have shown that LIX1 has a double-stranded RNA-binding domain, suggesting that it could be involved in mRNA or microRNA processing [10] and it has been shown that miR506 and miR-375 regulate YAP1 expression [30, 31]. It would thus be interesting to study whether LIX1 has a direct impact on YAP1/TEAD mRNA expression and/or stability.

We then demonstrated that LIX1 is an essential regulator of SMC differentiation. Intriguingly, while the proproliferative activity of LIX1 presumably facilitates SMC determination, LIX1 has a negative impact on further SMC differentiation. We suspect that high proliferative activity of LIX1 led to cell contact inhibition of proliferation, revealing the presence of a negative feedback loop on the endogenous expression and activity of YAP1 within the stomach mesenchyme to compensate for aberrant cell proliferation. Accordingly, we never observed hypertrophic stomachs under LIX1 influence, suggesting that LIX1 pro-proliferation activity is limited by the overall size of the stomach. In response to high cell density, the Hippo pathway regulates YAP1 activity through inhibitory phosphorylation [32] and we report here that the defect in SMC differentiation is associated with an increase in inactive phosphorylated YAP1 in LIX1-misexpressing stomachs. While the Hippo pathway has already been investigated in the context of gastrointestinal epithelia [28-30,33], our study is the first to suggest a role for this pathway in regulating the proliferation and differentiation of the gastrointestinal mesenchyme. Along these lines, the next step would be to address the possible regulation of the Hippo pathway by LIX1 in this developmental process. Lowfat, the arthropod homolog of LIX1, interacts with the atypical cadherins fat and dachsous and stabilizes FAT protein levels [12]. Although a recent study has shown that the vertebrate ortholog of FAT does not seem to regulate the Hippo pathway [34], FAT signalling has been shown to decrease YAP1 activity [35, 36]. One could thus speculate that, in the context of cell contact inhibition of proliferation, LIX1 participates more directly in the inhibition of YAP1 through the stabilization of FAT levels. Further investigations should focus on uncovering the potential molecular links that tie LIX1 to the regulation of YAP1 phosphorylation and transcriptional output.

Similarly to our conclusions for LIX1, we also report that while the pro-proliferative activity of YAP1 presumably facilitates SMC determination, it is sensitive to cell contact inhibition of proliferation and has a negative impact on further SMC differentiation. Because our misexpression experiments only led to mild overexpression of YAP1 (ranging from 1.2- to 3-fold), we speculate that the native stomach mesenchyme is poised to respond to mild over-activity of YAP1 by turning on the negative feedback loop on YAP1 activity. This finding contrasts with those of previous studies where high levels of YAP1 overexpression led to sustained proliferation and overgrowth of undifferentiated cells $[17,37]$. In any case, the compensatory mechanisms resulting from LIX1 or YAPI 
misexpression appeared to lock the determined mesenchyme in a state where the cells were neither proliferative nor differentiated. This state could simply reflect the requirement for a dynamic proliferation event between the determination and differentiation steps. By this hypothesis, because determined LIX1/YAP1-expressing cells are in contact inhibition of proliferation, differentiation could not be initiated. Alternatively, we could speculate that a certain level of YAP1 activity is necessary to initiate SMC differentiation, and because YAP1 activity has been turned off as a consequence of aberrant cell proliferation at the determination stage, differentiation could not be initiated. This second hypothesis highlights the possibility that YAP1 plays a dual role in regulating stomach mesenchyme progenitor development, both during the proliferative phase and later on during the differentiation phase. This hypothesis concords with emerging data showing that YAP1 regulates multiple signalling pathways, such as Wnt, BMP and Notch [38], and that Hippo signalling regulates Notch signalling [39]. Interestingly, all of these pathways are involved in the development of the GI tract [1, 6, 19, 40-42]. Further investigations are required to examine how YAP1 signalling is integrated in the regulation of SMC differentiation. YAP1 could be cooperating with two different transcription factors to regulate the processes of mesenchyme proliferation and SMC differentiation, similarly to what has recently been described during self-renewal of the intestinal epithelium [28]. In that system, the authors showed that YAP1 cooperates with Klf4 in promoting differentiation of intestinal goblet cells. Klf4 has been shown to abrogate the expression of myocardin, a major regulator of SMC differentiation [21], and of myocardin-induced expression of SMC genes [43], while YAP1 has been shown to interact with myocardin and interfere with its activity [13].

\section{Conclusion}

Altogether, our results demonstrate that LIX1 is a novel and unique marker of digestive mesenchyme immaturity and a regulator of mesenchymal progenitor proliferation and differentiation through its capacity to regulate YAP1 activity and density-dependent proliferation. Additionally, we demonstrate that this activity of LIX1 is conserved in cell culture, suggesting that the mechanism of LIX1 action outlined here is not limited to the developing stomach mesenchyme. In light of these conclusions, it would be interesting to investigate whether the activity of LIX1 is conserved throughout the more general context of organ size control and tissue regeneration. Finally, we have highlighted, through a developmental approach, three properties of LIX1 that could make it essential in cancer research. LIX1 defines an immature state of stomach smooth muscle, regulates cell proliferation within this immature mesenchyme and regulates the activity of the oncogene YAP1. These three properties thus point to the interest of further studies to examine the possible function of LIX1 in tumorigenesis and tumour progression.

\section{Methods \\ Chick embryonic GI tissues}

Fertilized White Leghorn eggs from the Haas Farm (France) were incubated at $38{ }^{\circ} \mathrm{C}$ in humidified incubators. Embryos were staged according to Hamburger and Hamilton [44]. Isolation of mesodermal and endodermal layers from stage 25 stomachs (referred to as E5) was performed as previously described [8]. The efficiency of dissections was evaluated by monitoring the expression of $S H H$ and BARX1, which are specific markers of the epithelial and mesenchymal layers, respectively.

\section{Avian retroviral misexpression system and constructs}

Chick LIX1 full-length cDNA was isolated from total mRNA extracts of E5 stomachs. The mouse YAP1, the chick full-length LIX1, the human full-length LIX1 and the Short hairpin RNA of LIX1 (Gallus target sequence: TCT TTG CAG CTG GTG ATT G, referred to as ShLIX1) associated with the mouse U6 promoter were cloned into the shuttle vector Slax13 and then subcloned into the Replication-Competent Avian Leucosis Sarcoma virus strain A (RCAS(A)) or strain B (RCAS(B)) vectors. Using RCAS vectors with two different envelopes (A and B) allows the introduction of two genes into a single cell [45]. FGF8, $s F G F R 2 b$ and GFP retroviral constructs have been previously described [8]. RCAS(A)-shPROX1 retrovirus [46] served as unrelated RCAS-ShRNA retroviruses. Retroviral constructs were transfected into the chicken DF-1 fibroblast cell line (ATCC-LGC) to produce retroviruses. Retroviruses were titered using standard techniques and injected into the splanchnopleural mesoderm of E1.5 chicken embryos to target the stomach mesenchyme [22]. Embryos were co-injected with RCAS-GFP to allow screening of correctly targeted stomachs. Eggs were then placed at $38{ }^{\circ} \mathrm{C}$ until harvested. Efficient retroviral infection was confirmed by in situ hybridization analysis on paraffin sections using $E N V$ probes or, in LIX1 misexpression experiments only, LIX1 probes. Infection with RCAS-GFP retroviruses does not affect chick stomach development (Additional file 10: Figure S9). Stomach phenotypes from infected embryos were analysed by comparison with uninfected control embryos incubated at the same time.

\section{Cell cultures and analysis}

The chicken DF-1 fibroblast cell line was cultured as previously described [22]. Cell growth in DF-1 cultures was assessed using the Muse Count and Viability reagent following the manufacturer's specifications (Muse Cell 
Analyzer - Millipore). DF-1 cells were plated on plastic at 2000 cells $/ \mathrm{cm}^{2}$ to obtain low-density cultures and $6000 \mathrm{cells} / \mathrm{cm}^{2}$ to obtain high-density cultures. Verteporfin (Sellekchem) was used applied to DF-1 cells for 20 hours at a final concentration of $1 \mu \mathrm{M}$.

\section{In situ hybridization and immunofluorescence staining}

Dissected GI tissues were fixed in $4 \%$ paraformaldehyde at room temperature for 30 minutes, washed in PBS, gradually dehydrated in methanol and stored at $-20{ }^{\circ} \mathrm{C}$ before processing for whole-mount in situ hybridization as previously described $[8,22]$. For sections, GI tissues were fixed in $4 \%$ paraformaldehyde at room temperature for 30 minutes, washed in PBS, gradually dehydrated in ethanol and embedded in paraffin. $10-\mu \mathrm{m}$ sections were cut using a microtome and collected on poly-L-lysine-coated slides (Thermo Fisher). Partial chick YAP1, CTGF and CYR61 cDNAs were isolated from total mRNA extracts of E5 stomachs. In situ hybridization experiments on whole-mount and paraffin sections were carried out as previously described [24] using chick LIX1 and YAP1 probes and published $S M 22, B A P X 1, S O X 10$ and $E N V$ probes $[8,19,24]$. Immunofluorescence studies were performed on paraffin sections using polyclonal antibodies against aSMA (Abcam Cat\# ab5694 RRID:AB_2223021 1:400 dilution), anti-Phospho-Histone H3-Ser10 (PH3) (Millipore Cat\# 06-570 RRID:AB_310177, 1:300 dilution), cleaved CASPASE-3 (Cell Signaling Technology Cat\# 9664S RRID:AB_331453, 1:400 dilution) and monoclonal antibodies against CALPONIN (Abnova Cat\# MAB1512 RRID:AB_1672405, 1:500 dilution). Nuclei were labelled with Hoechst (Invitrogen). In vivo proliferation rates were assessed by counting the number of PH3positive cells relative to the total number of nuclei in the section. Cell density was assessed on images of stomach sections by calculating the area occupied by Hoechst-stained nuclei relative to the total area of the section.

\section{Reverse transcription and quantitative polymerase chain reaction (RT-qPCR)}

Total RNA was extracted from stomachs or cell cultures with the HighPure RNA Isolation kit (Roche). Reverse transcription was performed using the Verso cDNA synthesis kit (Thermo Scientific) and RT-qPCR was performed using LightCycler technology (Roche Diagnostics). PCR primers (Additional file 11: Table S2) were designed using the LightCycler Probe Design 2.0 software. Each sample was analysed in three independent experiments done in triplicate. Expression levels were determined with the LightCycler analysis software (version 3.5) relative to standard curves. Data were represented as the mean level of gene expression relative to the expression of the reference genes UBIQUITIN or GAPDH. Relative mRNA expression was calculated using the $2^{-\Delta \Delta \mathrm{CT}}$ method [47].

\section{Western blotting}

DF-1 cells and chick stomachs were re-suspended in lysis buffer (20 mM Tris pH 8, $50 \mathrm{mM} \mathrm{NaCl}, 1 \% \mathrm{NP} 40$, cOmplete EDTA-free Protease Inhibitor Cocktail (Roche)); $10 \mu \mathrm{g}$ of total protein lysates were boiled in SDS-PAGE sample buffer, separated by $10 \%$ SDS-PAGE and transferred to nitrocellulose membranes. Membranes probed with rabbit anti-phospho-YAP (Ser127; Cell Signaling Technology Cat\# 4911S RRID:AB_2218913, 1:1000 dilution), anti-YAP/TAZ (Cell Signaling Technology Cat\# 8418S RRID:AB_10950494, 1:1000 dilution) or anti-GAPDH (SigmaAldrich Cat\# G9545 RRID:AB_796208, 1:5000 dilution) antibodies overnight. Glyceraldehyde-3-phosphate dehydrogenase (GAPDH) expression was used to confirm equal loading. All immunoblots were developed and quantified using the Odyssey infrared imaging system (LICOR Biosystems) and infrared-labelled secondary antibodies.

\section{Statistical analysis}

Data were analysed by performing two-tailed or, when appropriate, one-tailed Mann-Whitney tests using GraphPad Prism 6 software. Values of $\mathrm{n}$ represent the number of biological replicates. Each value used for statistical analyses is the mean of three technical replicates. Results were considered significant when $P<0.05\left(^{(*)}, P<0.01\left(^{(* *)}, P<0.001\right.\right.$ $(* * *)$ or $P<0.0001{ }^{(* * * *)}$.

\section{Photography}

Images were acquired using a Nikon Multizoom AZ100 stereomicroscope and a Carl Zeiss AxioImager microscope. Images presented in the figures are representative of the main phenotype observed in the population of infected embryos (Additional file 4: Table S1).

\section{Availability of data and materials}

Data supporting the results of this article are available in Additional file 12.

\section{Additional files}

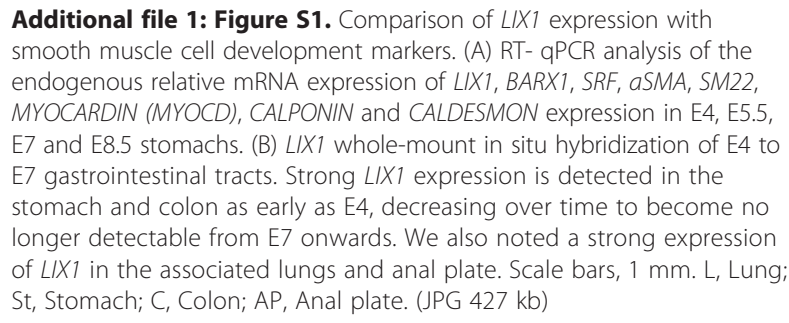

Additional file 2: Figure S2. RCAS retroviral infection. (A) Schematic representation of RCAS retroviral infection. RCAS(A)-ShLIX1 or RCAS(B)-LIX1 
retroviral particles are injected in the splanchnopleura, between somites 3 and 7 of embryos at E1.5. NT, Neural tube; NC, Notochord. (B) LIX1 whole-mount in situ hybridization on E7.5 control or LIX1-expressing stomachs. (JPG $460 \mathrm{~kb}$ )

Additional file 3: Figure S3. Specificity of the ShLIXI construct for $L I X 1$ mRNA. (A) Analysis of $L I X 1, a S M A$ and BARX1 expression by RT-GPCR in E6.5 control and RCAS-ShPROX1-expressing stomachs. Data were normalized to GAPDH expression. Normalized expression levels were converted to fold changes. Values are presented as the mean \pm standard error of the mean of $n=8$ controls vs. $n=8$ ShPROX1-expressing stomachs. ns, Not significant by one-tailed (for $L(X 1)$ or two-tailed (for aSMA and BARXI) Mann-Whitney tests. (B) Analysis of LIXI, aSMA and BARXI expression by RT-qPCR in E6.5 control stomachs, RCAS(A)-ShLIX1- expressing stomachs or RCAS(A)-ShLIX1/RCAS(B)-hLIX1-co-expressing stomachs. Data were normalized to GAPDH expression. Normalized expression levels were converted to fold changes. Values are presented as the mean \pm standard deviation of $n=12$ controls vs. $n=8$ Sh $L I X 1$ vs. $n=8$ Sh $L I X 1+h L I X 1$-expressing stomachs. ${ }^{*} P<0.05$ by one-tailed Mann-Whitney test. ns, Not significant. (JPG $170 \mathrm{~kb})$

Additional file 4: Table S1. Phenotype quantification. Quantification of embryos harbouring an abnormal stomach muscle phenotype, as demonstrated by in situ hybridization or immunostaining, following injection of RCAS(A)-ShLIXI (LIXI loss-of-function) or RCAS(B)-LIXI (LIX1 gain-of-function). (PDF $40 \mathrm{~kb}$ )

Additional file 5: Figure S4. Expression pattern of YAP1 and its transcriptional target genes during gastrointestinal development. (A) YAP1, CTGF, CYR61 and SM22 whole-mount in situ hybridization of E4-8 gastrointestinal tracts. L, Lung; St, Stomach; Co, Colon. (B) RT- aPCR analysis of the endogenous relative mRNA expression of YAP1, CTGF, CYR61 in E4-8.5 stomachs. (C) RT-qPCR analysis of the endogenous relative mRNA expression of YAP1, CYR61, CTGF, SHH, aSMA and BARX1 in mesenchymal and endodermal layers dissected from E5 stomachs. Total, Whole stomach; Epithelium, Epithelial layer; Mesenchyme, Mesenchymal layer. Values are presented as the mean \pm standard deviation of $n=3$ experiments. ${ }^{*} P<0.05$ by one-tailed Mann-Whitney test. ns, Not significant. Raw data for panel $C$ are shown in Additional file 12. (JPG $1455 \mathrm{~kb}$ )

Additional file 6: Figure S5. Sustained $L I X 1$ expression does not affect apoptosis. Transverse sections of E6 control and LIX1-expressing stomachs analysed by immunohistochemistry with anti-cleaved CASPASE-3 antibodies. Black arrows indicate the area imaged at high power in the cleaved-CASPASE-3 panels. Black arrowheads point to cleaved CASPASE-3-positive apoptotic cells. (JPG 402 kb)

Additional file 7: Figure S6. Sustained $L I X 1$ expression hinders smooth muscle cell differentiation. Analysis of CALPONIN and CALDESMON expression by RT-qPCR in E8.5 control GFP- and LIX1-expressing stomachs. Data were normalized to GAPDH expression. Normalized expression levels were converted to fold changes. Values are presented as the mean \pm standard deviation of $n=4$ controls vs. $n=5$ LIX 1 -expressing stomachs. ${ }^{* *} P<0.01$ by one-tailed Mann-Whitney test. ns, Not significant. Raw data are shown in Additional file 12. (JPG $91 \mathrm{~kb}$ )

Additional file 8: Figure S7. Sustained YAP1 expression hinders smooth muscle cell differentiation. (A) Serial transverse sections of E7 control GFP- and YAP1-expressing stomachs analysed either by in situ hybridization using the ENV riboprobe or by immunofluorescence with anti-aSMA and anti-CALPONIN antibodies. Nuclei were visualized with Hoechst. (B) Western blot analysis of YAP and phospho-YAP (72 kDa) levels in protein extracts from control GFP- and YAP1-expressing stomachs. Equal loading was verified by GAPDH expression $(37 \mathrm{kDa})$. Graph represents the quantification of western blot data. Normalized expression levels were converted to fold changes. Values are presented as the mean \pm standard deviation of $n=6$ controls vs. $n=6$ YAP1-expressing stomachs. ${ }^{*} P<0.05$ by one-tailed Mann-Whitney test. (C) RT-qPCR analysis of relative mRNA expression in E7 control GFP- and YAP1-expressing stomachs. Data were normalized to GAPDH expression. Normalized expression levels were converted to fold changes. Values are presented as the mean \pm standard deviation of $n=8$ controls vs. $n=6$ YAP1-expressing stomachs. ${ }^{*} P<0.05$ by one-tailed Mann-Whitney test. (JPG 2004 kb)

Additional file 9: Figure S8. The pro-proliferative effect of $L I X 1$ is abolished when DF-1 cells are seeded at high density. (A) RT-qPCR analysis of relative mRNA expression in DF-1 cells infected with RCAS(B)-GFP control or RCAS(B)-LIX1 plated at high density and harvested at day 1. Data were normalized to GAPDH expression. Normalized expression levels were converted to fold changes. Values are presented as the mean \pm standard deviation (SD) of $n=2$ experiments. Raw data for panel A are shown in Additional file 12. (B) Examination of proliferation in GFP-expressing cells (control) and LIX1-expressing cells. Graphs represent the quantification of PH3-positive cells. Normalized expression levels were converted to fold changes. Values are presented as the mean \pm SD of $n=7$ experiments. ns, Not significant by two-tailed Mann-Whitney test. (C) Western blot analysis of YAP and phospho-YAP $(72 \mathrm{kDa}$ ) levels in protein extracts from GFP- and LIX1-expressing cells. Equal loading was verified by GAPDH expression (37 kDa). Graph represents the quantification of western blot data. Normalized expression levels were converted to fold changes. Values are presented as the mean \pm SD of $n=7$. (JPG $721 \mathrm{~kb})$

Additional file 10: Figure S9. Infection with RCAS-GFP retroviruses does not affect chick stomach development and differentiation. (A) Whole-mount in situ hybridization of E6 uninfected (control) and GFP-expressing gastrointestinal tracts using SM22, BAPX1 and SOX10 riboprobes. Scale bars, $1 \mathrm{~mm}$. (B) Transverse sections of E7 control and GFP-expressing stomachs analysed either by in situ hybridization using the $L I X 1$ riboprobe or by immunofluorescence with anti-aSMA and anti-CALPONIN antibodies. Nuclei were visualized with Hoechst. (C) Serial transverse sections of E7 control and GFP-expressing stomachs analysed by immunofluorescence using anti-PH3 antibodies. Nuclei were visualized with Hoechst. Graph represents the quantification of PH3-positive cells. ns, Not significant. (JPG 1115 kb)

Additional file 11: Table S2. Gene-specific chick primers used for RT-qPCR. List of primer sequences used for transcript amplification by RT-PCR. (PDF $36 \mathrm{~kb}$ )

Additional file 12. Supporting data. (XLSX $46 \mathrm{~kb})$

\section{Competing interests}

The authors declare that they have no competing interests.

\section{Authors' contributions}

$J M, P d S B$ and SF designed the study. JM, DM and SF performed experiments. PdSB and SF obtained funding. JM, PdSB and SF contributed to writing and editing. All authors read and approved the final manuscript.

\section{Acknowledgments}

Research was supported by a Trampolin grant ( $\left.\mathrm{N}^{\circ} 15681\right)$ from the Association Française contre les Myopathies (AFM) to SF, a grant from AFM $\left(N^{\circ} 18766\right)$ to $\mathrm{PdSB}$ and the French Association for CIPO patients to PdSB. JM had a studentship from the Ministère de I'Education Nationale de I'Enseignement Supérieur et de la Recherche (MENESR). The authors thank Prof EN Olson for the mouse YAP1 construct, members of the "Development of visceral smooth muscle and associated pathologies" team of the INSERM U1046 for comments, Azzouz Charrabi and Valérie Scheuermann for technical assistance, and Prof Doyle McKey for critical reading of the manuscript.

\section{Received: 29 January 2016 Accepted: 18 April 2016}

Published online: 28 April 2016

\section{References}

1. Le Guen L, Marchal S, Faure S, de Santa Barbara P. Mesenchymal-epithelial interactions during digestive tract development and epithelial stem cell regeneration. Cell Mol Life Sci. 2015;72:3883-96.

2. Wallace AS, Burns AJ. Development of the enteric nervous system, smooth muscle and interstitial cells of Cajal in the human gastrointestinal tract. Cell Tissue Res. 2005:319:367-82.

3. Gabella G. Development of visceral smooth muscle. Results Probl Cell Differ. 2002:38:1-37.

4. Owens GK, Kumar MS, Wamhoff BR. Molecular regulation of vascular smooth muscle cell differentiation in development and disease. Physiol Rev. 2004;84:767-801.

5. Nguyen AT, Gomez D, Bell RD, Campbell JH, Clowes AW, Gabbiani G. Smooth muscle cell plasticity: fact or fiction? Circ Res. 2013;112:17-22. 
6. Notarnicola C, Rouleau C, Le Guen L, Virsolvy A, Richard S, Faure S, et al. The RNA-binding protein RBPMS2 regulates development of gastrointestinal smooth muscle. Gastroenterology. 2012;143:687-97.

7. Hapkova I, Skarda J, Rouleau C, Thys A, Notarnicola C, Janikova M, et al. High expression of the RNA-binding protein RBPMS2 in gastrointestinal stromal tumors. Exp Mol Pathol. 2013;94:314-21.

8. Le Guen L, Notarnicola C, de Santa Barbara P. Intermuscular tendons are essential for the development of vertebrate stomach. Development. 2009;136: 791-801.

9. Sagnol S, Yang Y, Bessin Y, Allemand F, Hapkova I, Notarnicola C, et al. Homodimerization of RBPMS2 through a new RRM-interaction motif is necessary to control smooth muscle plasticity. Nucleic Acids Res. 2014;42:10173-84.

10. Bando T, Hamada Y, Kurita K, Nakamura T, Mito T, Ohuchi H, et al. Lowfat, a mammalian Lix1 homologue, regulates leg size and growth under the Dachsous/Fat signaling pathway during tissue regeneration. Dev Dyn. 2011; 240:1440-53.

11. Swindell EC, Moeller C, Thaller C, Eichele G. Cloning and expression analysis of chicken Lix1, a founding member of a novel gene family. Mech Dev. 2011;109:405-8.

12. Mao Y, Kucuk B, Irvine KD. Drosophila lowfat, a novel modulator of Fat signaling. Development. 2009;136:3223-33.

13. Xie C, Guo Y, Zhu T, Zhang J, Ma PX, Chen YE. Yap1 protein regulates vascular smooth muscle cell phenotypic switch by interaction with myocardin. J Biol Chem. 2012;287:14598-605.

14. An Y, Kang Q, Zhao Y, Hu X, Li N. Lats2 modulates adipocyte proliferation and differentiation via hippo signaling. PLoS One. 2013;8:e72042.

15. Zhao B, Ye X, Yu J, Li L, Li W, Li S, et al. TEAD mediates YAP-dependent gene induction and growth control. Genes Dev. 2008;22:1962-71.

16. Zhao B, Li L, Lei Q, Guan KL. The Hippo-YAP pathway in organ size control and tumorigenesis: an updated version. Genes Dev. 2010;24:862-74.

17. Halder G, Johnson RL. Hippo signaling: growth control and beyond. Development. 2011;138:9-22

18. Choi MC, Ryu S, Hao R, Wang B, Kapur M, Fan CM, et al. HDAC4 promotes Pax7-dependent satellite cell activation and muscle regeneration. EMBO Rep. 2014;15:1175-83.

19. Faure S, McKey J, Sagnol S, de Santa Barbara P. Enteric neural crest cells regulate vertebrate stomach patterning and differentiation. Development. 2015;142:331-42.

20. Méricskay M, Blanc J, Tritsch E, et al. Inducible mouse model of chronic intestinal pseudo-obstruction by smooth muscle-specific inactivation of the SRF gene. Gastroenterology. 2007;133:1960.

21. Huang J, Wang T, Wright AC, Yang J, Zhou S, Li L, et al. Myocardin is required for maintenance of vascular and visceral smooth muscle homeostasis during postnatal development. Proc Natl Acad Sci U S A. 2015; 112:4447-52.

22. Moniot B, Biau S, Faure S, Nielsen CM, Berta P, Roberts DJ, et al. SOX9 specifies the pyloric sphincter epithelium through mesenchymal-epithelial signals. Development. 2004;131:3795-804.

23. Chan SW, Lim CJ, Chong YF, Pobbati AV, Huang C, Hong W. Hippo pathway-independent restriction of TAZ and YAP by angiomotin. J Biol Chem. 2011;286:7018-26.

24. Faure S, Georges M, McKey J, Sagnol S, de Santa Barbara P. Expression pattern of the homeotic gene Bapx1 during early chick gastrointestinal tract development. Gene Expr Patterns. 2013;13:287-92.

25. Mandler M, Neubuser A. FGF signaling is required for initiation of feather placode development. Development. 2004;131:3333-43.

26. Gumbiner BM, Kim NG. The Hippo-YAP signaling pathway and contact inhibition of growth. J Cell Sci. 2014;127:709-17.

27. Liu-Chittenden Y, Huang B, Shim JS, Chen Q, Lee SJ, Anders RA, et al. Genetic and pharmacological disruption of the TEAD-YAP complex suppresses the oncogenic activity of YAP. Genes Dev. 2012;26:1300-5.

28. Imajo M, Ebisuya M, Nishida E. Dual role of YAP and TAZ in renewal of the intestinal epithelium. Nat Cell Biol. 2015;17:7-19.

29. Yu FX, Meng Z, Plouffe SW, Guan KL. Hippo pathway regulation of gastrointestinal tissues. Annu Rev Physiol. 2015;77:201-27.

30. Zhang ZW, Men T, Feng RC, Li YC, Zhou D, Teng CB. miR-375 inhibits proliferation of mouse pancreatic progenitor cells by targeting YAP1. Cell Physiol Biochem. 2013;32:1808-17.

31. Deng J, Lei W, Xiang X, Zhang L, Yu F, Chen J, et al. MicroRNA-506 inhibits gastric cancer proliferation and invasion by directly targeting Yap1. Tumour Biol. 2015;36:6823-31.
32. Zhao B, Wei X, Li W, Udan RS, Yang Q, Kim J, et al. Inactivation of YAP oncoprotein by the Hippo pathway is involved in cell contact inhibition and tissue growth control. Genes Dev. 2007;21:2747-61.

33. Zhou D, Zhang $Y$, Wu H, Barry E, Yin $Y$, Lawrence $E$, et al. Mst1 and Mst2 protein kinases restrain intestinal stem cell proliferation and colonic tumorigenesis by inhibition of Yes-associated protein (Yap) overabundance. Proc Natl Acad Sci U S A. 2011;108:E1312-20.

34. Bossuyt W, Chen CL, Chen Q, Sudol M, McNeill H, Pan D, et al. An evolutionary shift in the regulation of the Hippo pathway between mice and flies. Oncogene. 2014;33:1218-28.

35. Van Hateren NJ, Das RM, Hautbergue GM, Borycki AG, Placzek M, Wilson SA. FatJ acts via the Hippo mediator Yap1 to restrict the size of neural progenitor cell pools. Development. 2011;138:1893-902.

36. Ito T, Taniguchi H, Fukagai K, Okamuro S, Kobayashi A. Inhibitory mechanism of FAT4 gene expression in response to actin dynamics during Src-induced carcinogenesis. PLoS One. 2015;10:e0118336.

37. Camargo FD, Gokhale S, Johnnidis JB, Fu D, Bell GW, Jaenisch R, et al. YAP1 increases organ size and expands undifferentiated progenitor cells. Curr Biol. 2007;17:2054-60.

38. Hansen CG, Moroishi T, Guan KL. YAP and TAZ: a nexus for Hippo signaling and beyond. Trends Cell Biol. 2015;25:499-513.

39. Manderfield LJ, Aghajanian H, Engleka KA, Lim LY, Liu F, Jain R, et al. Hippo signaling is required for Notch-dependent smooth muscle differentiation of neural crest. Development. 2015;142:2962-71.

40. Smith DM, Nielsen C, Tabin CJ, Roberts DJ. Roles of BMP signaling and Nkx2.5 in patterning at the chick midgut-foregut boundary. Development. 2000;127:3671-81.

41. Theodosiou NA, Tabin CJ. Wnt signaling during development of the gastrointestinal tract. Dev Biol. 2003;259:258-71.

42. de Santa BP, Williams J, Goldstein AM, Doyle AM, Nielsen C, Winfield S, et al. Bone morphogenetic protein signaling pathway plays multiple roles during gastrointestinal tract development. Dev Dyn. 2005;234:312-22.

43. Liu Y, Sinha S, McDonald OG, Shang Y, Hoofnagle MH, Owens GK. Kruppellike factor 4 abrogates myocardin-induced activation of smooth muscle gene expression. J Biol Chem. 2005;280:9719-27.

44. Hamburger $\mathrm{V}$, Hamilton HL. A series of normal stages in the development of the chick embryo. J Morph. 1951;88:49-92.

45. Givol I, Tsarfaty I, Resau J, Rulong S, da Silva PP, et al. Bcl-2 expressed using a retroviral vector is localized primarily in the nuclear membrane and the endoplasmic reticulum of chicken embryo fibroblasts. Cell Growth Differ. 1994:4:419-29.

46. Holzmann J, Hennchen M, Rohrer H. Prox1 identifies proliferating neuroblasts and nascent neurons during neurogenesis in sympathetic ganglia. Dev Neurobiol. 2015;75:1352-67.

47. Livak KJ, Schmittgen TD. Analysis of relative gene expression data using real-time quantitative PCR and the 2(-Delta Delta $C(T)$ ) method. Methods. 2001;25:402-8.

\section{Submit your next manuscript to BioMed Central and we will help you at every step:}

- We accept pre-submission inquiries

- Our selector tool helps you to find the most relevant journal

- We provide round the clock customer support

- Convenient online submission

- Thorough peer review

- Inclusion in PubMed and all major indexing services

- Maximum visibility for your research

Submit your manuscript at www.biomedcentral.com/submit
C Biomed Central 